\title{
Airway Tree Segmentation from CT Scans Using Gradient-Guided 3D Region Growing
}

\author{
Anna Fabijańska, Marcin Janaszewski, Michał Postolski, and Laurent Babout \\ Computer Engineering Department, Technical University of Lodz \\ 18/22 Stefanowskiego Str., 90-924, Lodz, Poland \\ \{an_fab, janasz, mpostol, lbabout\}@kis.p.lodz.pl
}

\begin{abstract}
In this paper a new approach to CT based investigation of pulmonary airways is introduced. Especially a new - fully automated algorithm for airway tree segmentation is proposed. The algorithm is based on $3 \mathrm{D}$ seeded region growing. However in opposite to traditional approaches region growing is applied twice: firstly - for detecting main bronchi, secondly - for localizing low order parts of the airway tree. The growth of distal parts of the airway tree is driven by a map constructed on the basis of morphological gradient.
\end{abstract}

Keywords: CT, airway tree, image segmentation, 3D region growing.

\section{Introduction}

CT chest scans are commonly used for investigation of pulmonary disorders, especially chronic obstructive pulmonary disease (COPD) which is a common name for pathological changes characterized by airflow limitation due to different combinations of airway disease and emphysema [1][2]. The thickness of an airway wall and diameter of an airway lumen can provide important information about many pulmonary diseases therefore identification of airways in CT scans is an important step for many clinical applications and for physiological studies [2].

Traditionally, analysis of chest CT scans was preformed by radiologists who recognized areas of abnormal airway properties in consecutive slices of the examined scan. However, analyzing about 400 slices covering chest area is too cumbersome for everyday clinical use. Moreover analysis performed manually resulted in subjective and qualitative estimation of airway abnormalities without accurate quantification of pathological changes.

The main goal of recent clinical applications is to provide a useful tool for characterizing airway data. The major challenge of such applications is airway tree segmentation from CT scans. It is very difficult due to inhomogenity of a bronchial lumen, adjacency of the blood vessels, and changes of grey levels along airway walls.

In this paper problem of airway tree reconstruction from volumetric CT data is considered. Especially, segmentation using modified, 3D region growing method is regarded. In the proposed approach the growth of an airway tree is guided and constrained by morphological gradient. 


\section{Airway Tree}

An airway tree is a part of respiratory tract that conducts air into the lungs. It starts with trachea which splits into two main bronchi: the left and the right one. The main bronchi subdivide into two segmental bronchi. These in turn split into twigs called bronchioles. Divisions of the airways into the smaller ones define orders of bronchi.

An illustrative image of the airway tree is presented in Figure 1. Consecutive numbers denote trachea (1), left (2a) and right main bronchus (2b), bronchi (3) and bronchioles (4). All branches of the airway tree are composed from airway lumen surrounded by high-density vascular airway wall. An airway lumen is filled with air.

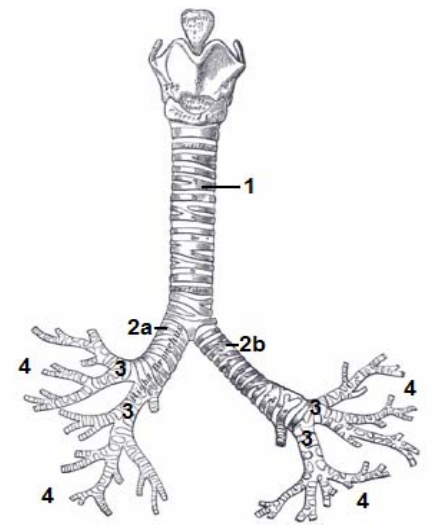

Fig. 1. 3D view of an airway tree; 1 - trachea; $2 \mathrm{a}$ - right main bronchus; $2 \mathrm{~b}-$ left main bronchus; 3 - bronchi; 4 - bronchioles

\section{Related Works}

Different approaches to airway tree segmentation have been reported in the literature so far. However, in general they can be qualified as 3D approaches or 3D/2D (hybrid) approaches.

Three-dimensional approaches to airway tree segmentation act on a 3D volumetric image build up from series of planar CT images combined into a stack. Mostly, they involve seeded 3D region-growing supported by 3D propagation procedures such as combination of axial and radial propagation potentials [4] or non linear filters scanning for short airway sections [5] and connecting branch segments sharing common walls [6]. Up to 5 orders of bronchi could be detected using this method.

Hybrid techniques for airway tree reconstruction combine planar analysis with 3D segmentation. Usually, conventional 3D region-growing is firstly used to identify large airways. Secondly 2D analysis of consecutive CT slices is performed. It aims to define potential localization of candidate airways in planar images. Algorithms of candidate airways detection involve various techniques. The most important ones are:

- Rule-based techniques which utilize anatomical knowledge about airways and blood vessels. Candidate airways are recognized on individual slices based on 
a priori information about airways and vessels [7][8]. These techniques often suffer from large numbers of falsely detected airways.

- Gray-level morphological techniques which use grayscale morphological reconstruction to identify local extremes in the image. Candidate for airways and vessels on consecutive CT slices are considered as valleys and peaks in the grayscale profile of the current slice [9][10].

- Wave front propagation techniques which start from already detected airways and propagate waves in 2D plane to detect walls of the bronchi [11][12].

- Template matching techniques that search consecutive slices for oval dark rings surrounding brighter areas (airways) [12] or dark solid oval areas (adjacent blood vessels). Templates may be predefined a priori [13] or set adaptively [12].

Few 2D approaches to airway tree investigation were also reported. In these methods only planar analysis of consecutive CT slices is carried out [2]. However, 2D approaches are in minority because of their poor efficiency and low accuracy.

Having in mind classification presented above introduced method can be considered as a tree dimensional one. It extracts up to 8-9 generations of bronchi while avoiding leakages and falsely detected branches.

\section{Input Data}

3D volumetric CT chest scans of several patients were examined. They were obtained from GE LightSpeed VCT Scanner. The average number of transverse slices per each examination was 450 with the slice thickness equal to $0.625 \mathrm{~mm}$. The slices were provided with 16-bit resolution and stored as signed 16-bit monochromatic images of the resolution $512 \times 512$ pixels. Individual slices were stacked into a 3D space representing volumetric data set. Exemplary CT slice with anatomical areas marked is presented in Figure 2.

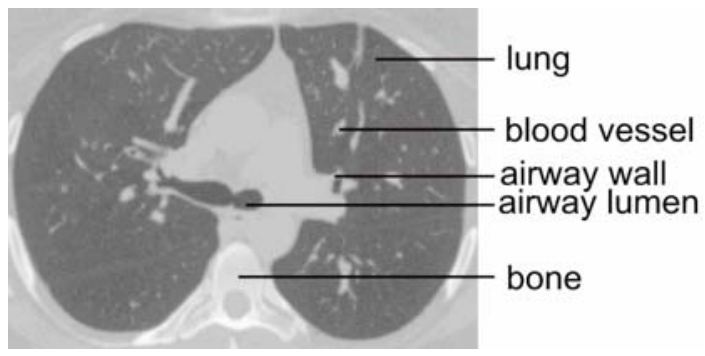

Fig. 2. Exemplary CT slice at the carina level with important anatomical areas marked

In analyzed images gray levels measured in Hunfield units (HU) ranged from 1024 to around 700. Grey levels which represent airway lumen and lung tissues filled with air were over $-1024 \mathrm{HU}$ but less than $-850 \mathrm{HU}$. Airway walls, blood vessels and other high-density vascular tissues were areas of the intensities within the range 300:50 HU. Intensities over $300 \mathrm{HU}$ matched hard tissues (bones). 


\section{Airway Tree Segmentation}

\subsection{D Seeded Region Growing - The Main Idea}

3D seeded region growing is a simple and convenient algorithm for image segmentation. In case of CT chest scans, the method starts from the seed point in the centre of trachea and builds a set of voxels by iteratively joining new similar pixels. In this way the whole airway tree should be segmented. This idea is presented on Figure 3a. However, as it was mentioned many times in the trade literature $3 \mathrm{D}$ region growing is not sufficient for segmentation of complete airway tree from CT scans due to its proneness to leakages.
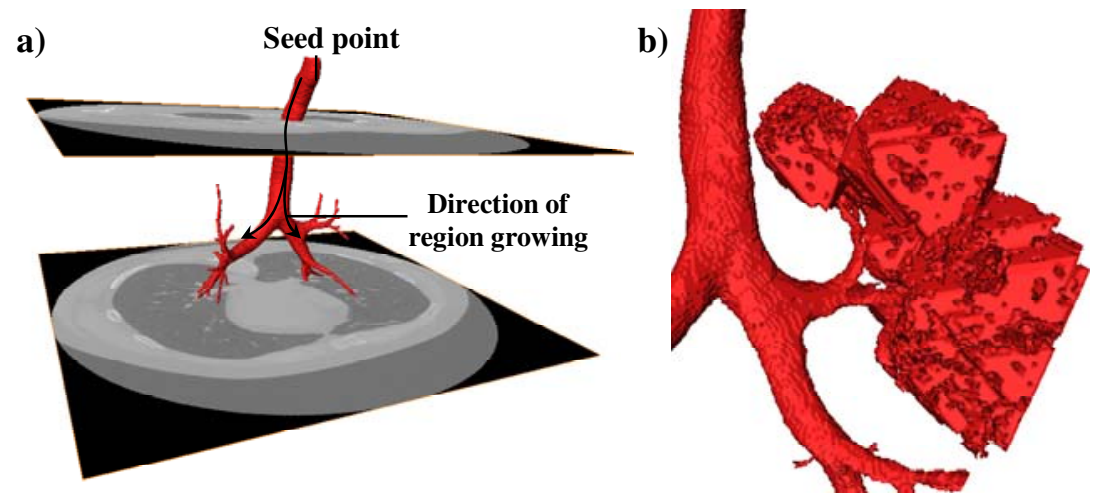

Fig. 3. Airway tree segmentation using seeded 3D region growing algorithm; a) the main idea; b) algorithm leaking into the lung parenchyma

On CT slices airway lumen is separated from surrounding lung parenchyma by a wall composed from high-density vascular tissue. Due to the difference in tissue densities, an airway wall appears significantly brighter than the airway lumen and lung tissues. However airway lumen and lung parenchyma are both filled with air therefore on CT scan they appear as areas of very similar grey levels. In consequence only one pixel of airway wall discontinuousness causes the 3D region growing algorithm to leak into the lung area (see Fig. 3b). In practice broken airway walls appear frequently in case of lower order bronchi. It is caused by imperfections of imaging devices which results in loss of spatial resolution and increasing noxious influence of the noise content. Therefore it is almost impossible to segment whole airway tree with simple region growing and avoid the leakage.

\subsection{Proposed Idea}

The proposed airway tree segmentation algorithm, as most approaches to the considered problem, is based on seeded 3D region growing. However, during airway lumen segmentation region growing is applied twice. Moreover, leak prevention mechanism is applied in order to avoid the algorithm to consider voxels that are part of the lung parenchyma. Successive steps of the algorithm are as follows: 
1. Image preprocessing.

2. Detection of main bronchi up to 5-6th divisions of airway tree using 3D seeded region growing.

3. Construction of a map defining possible locations of distal airways.

4. Detection of lower order bronchi using 3D region growing starting from previously detected airway tree and guided by the map of possible airway locations.

\section{Step 1: Image Preprocessing}

Before the main processing is applied, input CT data is smoothed in order to close broken airway walls and avoid leakages into the lungs. For data smoothing 3D median filter is applied. Traditional cubic $(3 \times 3 \times 3)$ mask is used in this stage.

It should be underlined, that smoothing helps to avoid leakages but also compromises details by removing important airway data - especially airways of dimensions comparable with the kernel of the smoothing filter are cleared out.

\section{Step 2: First Pass of 3D Region Growing}

3D seeded region growing is applied for the first time on median-smoothed CT data. The seed is automatically defined on the first slice of the data set as a voxel located in the centre of oval area of the trachea. Applied in this stage 3D region growing algorithm classifies the current voxel as voxel belonging to airway lumen if both of the following constrains are true:

- intensity of the current voxel differs from the average intensity of voxels classified to airway lumen not more than $T \%$;

- intensities of all 6 closest (connected) neighbours of the current voxel differ from the average intensity of voxels classified to airway lumen not more than $T \%$.

Value of $T$ is determined automatically on the first run. It equals to the lowest value for which region growing starts up. Then $T$ is decreased by half after trachea is segmented in order to avoid leakages into the lungs. At this stage of airway tree segmentation up to 5-6th orders of bronchi can be detected.

Results of the first pass of region growing applied to two exemplary CT data sets are presented in Figure 4.
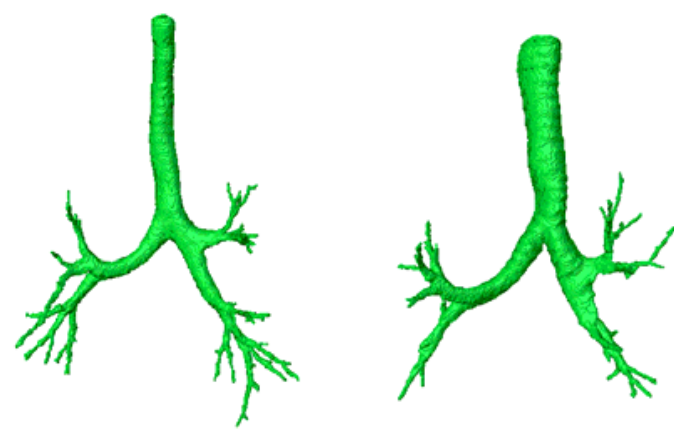

Fig. 4. Results of the first pass of region growing applied to two exemplary CT data sets 
Step 3: Construction of a map defining possible airway locations

In the following step of the airway tree segmentation 3D morphological gradient is calculated based on non-smoothed CT data. This transformation (which is a difference between results of $3 \mathrm{D}$ greyscale dilatation and $3 \mathrm{D}$ greyscale erosion) highlights sharp grey levels transitions connected also (but not only) with airway walls. Areas of the highest gradient which are connected with airway tree built in the previous step of the algorithm define possible locations of the distal bronchi.

In order to define map of the possible candidate airways, gradient image is firstly thresholded and then eroded with small structural element. Airways are supposed to be located in those binary areas which are connected with airway tree built in the previous step. For segmentation (which defines the areas of the highest gradient) the authors applied thresholding with local iterative threshold selection [14].

It should be underlined that morphological gradient after thresholding is to define possible location of the distal bronchi. Therefore in order to avoid loss of important bronchi information the transformation should be performed on non-smoothed image.

\section{Step 4: Second Pass of 3D Region Growing}

The last step of the airway tree segmentation is a second application of the 3D region growing algorithm. This time however it is performed on the original (non-smoothed) CT data. The algorithm starts from previously segmented airway tree and is guided by the map constructed on the basis of morphological gradient. Successive voxels are joined to airway tree if both of the following constrains are fulfilled:

- current voxel is situated in the area defined by the map constructed on the basis of morphological gradient;

- intensity of the current voxel differs from the average intensity of voxels classified to airway lumen not more than $2 T \%$.

Value of $T$ is remembered from the second step of the algorithm and changes during algorithm performance in the way as during the first pass of region growing.

\section{Results and Discussion}

Results of complete airway tree segmentation procedure applied to previously used CT data sets are presented in Figure 5. Airways detected during the second application of region growing are marked in red color. The green color represents fragments of bronchial tree extracted using the first region growing step.

The assessment of the proposed algorithm was made by comparison of obtained airway trees with the corresponding templates. The templates were obtained by skilled radiologist who marked and filled airway lumen areas manually in successive slices of analyzed CT scans. Figure 6 presents the comparison between exemplary airway trees from Figure 5, which are representative of all tested data sets and the corresponding templates. Missing airways are marked in red color. Green color corresponds to the tree obtained using proposed algorithm.

One can see from Figure 6 that the proposed algorithm of airway tree segmentation enables to extract up to 8-9 generations of bronchi. Falsely detected airways characteristic for knowledge-based methods [7][8] do not appear at all. Moreover guided and constrained growth of an airway tree excludes possibility of leakages into the lungs. 

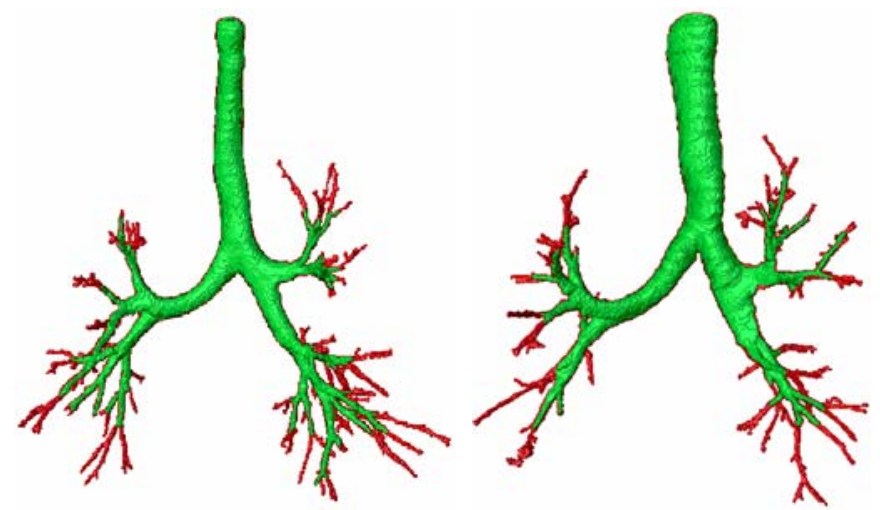

Fig. 5. Results of complete airway tree segmentation. Airways detected during second application of region growing are marked with red color.
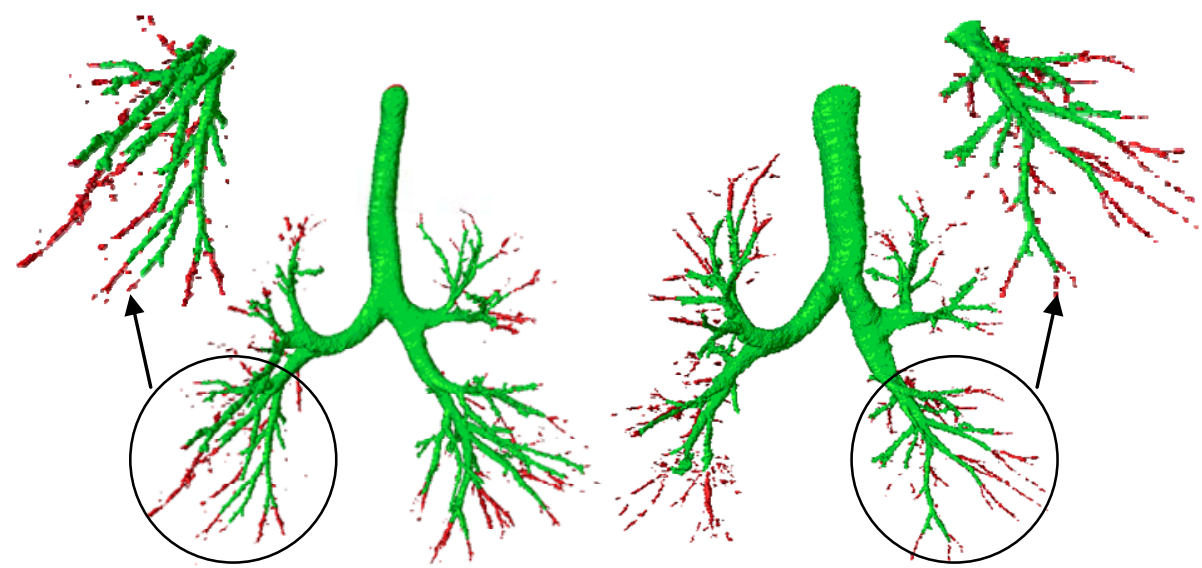

Fig. 6. Comparison of airway trees from Figure 5 with the corresponding templates. Missing airways are marked with red color.

The comparison between obtained airway trees and corresponding templates reveals that not more than one division of the bronchial tree was missed by the proposed algorithm. This means that information about 10 bronchi generations was present in considered CT data. This result is consistent with analysis presented in [9]. Having this in mind, the results presented in this paper can be considered interesting and accurate enough for further quantitative analysis of airway pathologies.

\section{Conclusions}

In this paper the problem of airway tree segmentation from CT chest scans was investigated. Especially fully automated algorithm for airway tree segmentation was introduced. The algorithm is based on a 3D approach which extracts airway trees from 
volumetric CT chest scans using region growing method guided and constrained by a morphological gradient. Such method allows to prevent leakages into the lungs and avoid falsely detected branches. Consequently, the proposed algorithm detects up to 9 generations of bronchi.

Nowadays, advanced CT scanners are able to resolve up to 10 orders of bronchial tree divisions in chest scans. Having this in mind the results obtained using the introduced algorithm can be considered interesting and accurate enough for further quantitative analysis of airway properties and its pathological changes.

\section{References}

1. American Thoracic Society: Standards for the diagnosis and care of patients with chronic obstructive pulmonary disease. Am. J. Resp. Crit. Care Med. 152, S77-S121 (1995)

2. Berger, P., Perot, V., Desbarats, P., Tunon-de-Lara, J.M., Marthan, R., Laurent, F.: Airway wall thickness in cigarette smokers: quantitative thin-section CT assessment. Radiology 235(3), 1055-1064 (2005)

3. Reilly, J.: Using computed tomographic scanning to advance understanding of chronic obstructive pulmonary disease. Proc. Am. Thorac. Soc. 3(5), 450-455 (2006)

4. Felita, C., Prêteux, F., Beigelman-Aubry, C., Grenier, P.: Pulmonary airways: 3-D reconstruction from multislice CT and clinical investigation. IEEE Trans. Med. Imag. 23(11), 1353-1364 (2004)

5. Graham, M., Gibbs, J., Higgins, W.: Robust system for human airway-tree segmentation. In: Proc. SPIE, vol. 6914, pp. 69141J-1 - 69141J-18 (2008)

6. Busayarat, S., Zrimec, T.: Detection of bronchopulmonary segments on high-resolution CT-preliminary results. In: 20th IEEE Int. Symp. Computer-Based Medical Systems, pp. 199-204 (2007)

7. Sonka, M., Park, W., Hoffman, E.: Rule-based detection of intrathoracic airway trees. IEEE Trans. Med. Imag. 15(3), 314-326 (1996)

8. Brown, M., McNitt, M., Mankovich, N., Goldin, J., Aberle, D.: Knowledge-based automated technique for measuring total lung volume from CT. In: Proc. SPIE, vol. 2709, pp. 63-74 (1996)

9. Aykac, D., Hoffman, E., McLennan, G., Reinhardt, J.: Segmentation and analysis of the human airway tree from three-dimensional X-ray CT images. IEEE Trans. Med. Imag. 22(8), 940-950 (2003)

10. Pisupati, C., Wolf, L., Mitzner, W., Zerhouni, E.: Segmentation of 3D pulmonary trees using mathematical morphology. In: Mathematical morphology and its applications to image and signal processing, pp. 409-416. Kluwer Academic Publishers, Dordrecht (1996)

11. Wood, S., Zerhouni, A., Hoffman, E., Mitzner, W.: Measurement of three-dimensional lung tree structures using computed tomography. J. Appl. Physiol. 79(5), 1687-1697 (1995)

12. Mayer, D., Bartz, D., Ley, S., Thust, S., Heussel, C., Kauczor, H., Straßer, W.: Segmentation and virtual exploration of tracheobronchial trees. In: 17th Int. Cong. and Exhibition Computer Aided Radiology and Surgery, London, UK, pp. 35-40 (2003)

13. Chabat, F., Xiao-Peng, H., Hansell, D., Guang-Zhong, Y.: ERS transform for the automated detection of bronchial abnormalities on CT of the lungs. IEEE Trans. Med. Imag. 20(9), 942-952 (2001)

14. Strzecha, K., Fabijańska, A., Sankowski, D.: Segmentation algorithms for industrial image quantitative analysis system. In: 17th IMEKO World Congress Metrology for a Sustainable Development, p. 164. Rio de Janeiro, Brazil (2006) 